\title{
Correction to: Curcumin suppressed proliferation and migration of human retinoblastoma cells through modulating NF- $\mathrm{KB}$ pathway
}

\author{
Ying-Tao Mu $\cdot$ Huan-Huan Feng $\cdot$ Jin-Qiang Yu $\cdot$ Zhen-Kai Liu \\ Yan Wang $\cdot$ Jie Shao $\cdot$ Ran-Hui Li $\cdot$ De-Kun Li $\mathbb{C}$
}

Published online: 4 May 2021

(C) Springer Nature B.V. 2021

\section{Correction to:}

Int Ophthalmol (2020) 40:2435-2440

https://doi.org/10.1007/s10792-020-01406-4

In the original publication, the affiliation of authors Ying-Tao Mu, Jin-Qiang Yu, Zhen-Kai Liu, Yan Wang, Jie Shao and De-Kun Li were published incorrectly. The correct affiliation is given in this correction.

Publisher's Note Springer Nature remains neutral with regard to jurisdictional claims in published maps and institutional affiliations.

The original article can be found online at https:// doi.org/10.1007/s10792-020-01406-4.

\section{Y.-T. Mu}

Department of TCM, Renmin Hospital, Hubei University of Medicine, Shiyan 442000, Hubei, China

\section{H.-H. Feng}

Xiangyang No.1 People's Hospital, Hubei University of Medicine, Xiangyang, Hubei, China

J.-Q. Yu · Z.-K. Liu · Y. Wang · J. Shao · D.-K. Li (ه) Department of Ophthalmology, Renmin Hospital, University of Medicine, Shiyan, China

e-mail: 36773378@qq.com

R.-H. Li

Institute of Pathogen Biology, University of South China, Heyang, Hunan, China 